\title{
The Order Completion Method for Systems of Nonlinear PDEs: Pseudo- topological Perspectives
}

\author{
Jan Harm van der Walt \\ Department of Mathematics and Applied Mathematics \\ University of Pretoria
}

\begin{abstract}
By setting up appropriate uniform convergence structures, we are able to reformulate the Order Completion Method of Oberguggenberger and Rosinger in a setting that more closely resembles the usual topological constructions for solving PDEs. As an application, we obtain existence and uniqueness results for the solutions of arbitrary continuous, nonlinear PDEs.
\end{abstract}

Keywords: Nonlinear PDEs, Order Completion, Uniform Convergence Space

Mathematics Subject Clasifications (2000): 34A34, 54A20, 06B30, 46E05

\section{Introduction and Preliminary Remarks}

\subsection{General, Type Independent Theories for PDEs}

It is widely believed that there is no general, type independent theory concerning existence and regularity of solutions to arbitrary nonlinear PDEs. Indeed, the book 'Lecture notes on PDEs' by I. V. Arnold (Arnold, 2004) starts with the following remark:

"In contrast to ordinary differential equations, there is no unified theory of partial differential equations. Some equations have their own theories, while others have no theory at all. The reason for this complexity is a more complicated geometry..."

Within the confines of functional analysis, and more broadly speaking, general topology, this was until very recently the case. However, within the last fifteen years, two such general, type independent theories for the solutions PDEs appeared.

In 1994 there appeared a type independent theory of existence and regularity of solutions to nonlinear systems of PDEs based on order completion of sets of functions, see (Oberguggenberger and Rosinger, 1994). This theory yields the existence, and uniqueness, of solutions to arbitrary continuous and nonlinear systems of PDEs, with the solutions assimilated with Hausdorff continuous functions, see (Anguelov and Rosinger, 2005).

Also, within the confines of functional analysis, there recently emerged a general and type independent theory of PDEs, see (Neuberger, 1997) through (Neuberger, 2005). This theory, developed by Neuberger, is based on approximations within Hilbert space obtained by a generalized method of Steepest Descent. This method has yielded exceptional numerical results.

Both methods are in fact able to solve equations that are far more general than PDEs. This is precisely the feature which makes them so powerful and type independent when applied to the particular case of PDEs.

\subsection{A Brief Overview of the Order Completion Method}

Let us recall the general construction for solving a PDE of the form

$$
T(x, D) u(x)=f(x), x \in \Omega
$$


$\mathrm{J} \mathrm{H}$ van der Walt

by topological methods. Here $\Omega \subseteq \mathbb{R}^{n}$ is supposed to be nonempty and open. The right hand term $f$ is assumed continuous, and the unknown function satisfies, initially at least, $u \in \mathcal{C}^{m}(\Omega)$. The operator $T(x, D)$ is supposed to be defined by some continuous function $F: \Omega \times \mathbb{R}^{M} \rightarrow \mathbb{R}$ through

$$
T(x, D) u(x)=F\left(x, u(x), \ldots, D^{\alpha} u(x), \ldots\right), x \in \Omega \text { and }|\alpha| \leq m
$$

The general construction for finding generalized solutions to (1) through (2), within the context of topology, and specifically functional analysis, is summarized as follows:

(1) Start with a PDE-operator $T$ mapping some initial space of classical functions $X$ into some space of functions $Y$, with the righthand term $f \in Y$.

(2) Define some structure, e.g. a uniformity, on $Y$.

(3) Define a structure of the same sort on $X$ through pullback of the operator $T$ to obtain a space $X_{T}$ so that $T$ is compatible with the customary structures on $X$ and $Y$.

(4) Construct the completions of $X_{T}$ and $Y$, say $X_{T}^{\sharp}$ and $Y^{\sharp}$, and extend $T$ to a mapping $T^{\sharp}: X_{T}^{\sharp} \rightarrow Y^{\sharp}$

(5) If $f \in T^{\sharp}\left(X_{T}^{\sharp}\right)$, one obtains a generalized solution to the equation

The order completion method operates in a similar fashion, see (Oberguggenberger and Rosinger, 1994). It is based on the fundamental approximation result that, under a condition which is necessary for the existence of a classical solution at $x \in \Omega$, every continuous righthand term $f$ in (1) can be approximated from below (or from above) by functions in $\mathcal{C}_{n d}^{m}(\Omega)$ where

$$
f: \Omega \rightarrow \mathbb{R} \in \mathcal{C}_{n d}^{m}(\Omega) \Leftrightarrow\left(\begin{array}{l}
\exists \Gamma_{f} \subset \Omega \text { closed nowhere dense : } \\
f \in \mathcal{C}^{m}\left(\Omega \backslash \Gamma_{f}\right)
\end{array}\right)
$$

In this regard, for any $f \in \mathcal{C}^{0}(\Omega)$ we have

$$
\begin{aligned}
& \forall \epsilon>0: \\
& \exists \Gamma_{\epsilon} \subset \Omega \text { closed nowhere dense, } U_{\epsilon} \in \mathcal{C}^{m}\left(\Omega \backslash \Gamma_{\epsilon}\right): \\
& f(x)-\epsilon \leq T(x, D) U_{\epsilon}(x) \leq f(x), x \in \Omega \backslash \Gamma_{\epsilon}
\end{aligned}
$$

Essentially, what this means is that the image of $\mathcal{C}_{n d}^{m}(\Omega)$ under $T(x, D)$ is order dense in $\mathcal{C}_{n d}^{0}(\Omega)$.

One now proceeds as follows: On the set $\mathcal{C}_{n d}^{0}(\Omega)$ one considers the equivalence relation

$$
f \sim g \Leftrightarrow\left(\begin{array}{l}
\exists \Gamma_{f, g} \subset \Omega \text { closed, nowhere dense : } \\
x \in \Omega \backslash \Gamma_{f, g} \Rightarrow f(x)=g(x)
\end{array}\right)
$$

On the quotient space $\mathcal{M}^{0}(\Omega)=\mathcal{C}_{n d}^{0}(\Omega) / \sim$, one introduces the partial order

$$
F \leq G \Leftrightarrow\left(\begin{array}{l}
\exists f \in F, g \in G, \Gamma \subset \Omega, \text { closed, nowhere dense : } \\
(1) f, g \in \mathcal{C}^{0}(\Omega \backslash \Gamma) \\
(2) f \leq g \text { on } \Omega \backslash \Gamma
\end{array}\right)
$$

By the continuity of the operator $T(x, D)$ it follows that if $u \in \mathcal{C}_{n d}^{m}(\Omega)$, and $u \in \mathcal{C}^{m}(\Omega \backslash \Gamma)$, then $T(x, D) u \in \mathcal{C}^{0}(\Omega \backslash \Gamma)$ so that one has the well defined mapping

$$
T(x, D): \mathcal{C}_{n d}^{m}(\Omega) \rightarrow \mathcal{C}_{n d}^{0}(\Omega)
$$


On the set $\mathcal{C}_{n d}^{m}(\Omega)$ one considers the equivalence relation induced by the PDE operator $T(x, D)$

$$
u \sim_{T} v \Leftrightarrow T(x, D) u \sim T(x, D) v .
$$

With the mapping (7) one can now associate in a canonical way an injective mapping

$$
T: \mathcal{M}_{T}^{m}(\Omega) \rightarrow \mathcal{M}^{0}(\Omega),
$$

where $\mathcal{M}_{T}^{m}(\Omega)=\mathcal{C}_{n d}^{m}(\Omega) / \sim_{T}$. Next one introduces a partial order on $\mathcal{M}_{T}^{m}(\Omega)$ by

$$
U \leq_{T} V \Leftrightarrow T U \leq T V \text { in } \mathcal{M}^{0}(\Omega)
$$

This construction results in an order isomorphic embedding

$$
T: \mathcal{M}_{T}^{m}(\Omega) \hookrightarrow \mathcal{M}^{0}(\Omega) .
$$

By extension of $T$ to the Dedekind completion $\mathcal{M}_{T}^{m}(\Omega)^{\sharp}$ one obtains the following commutative diagram

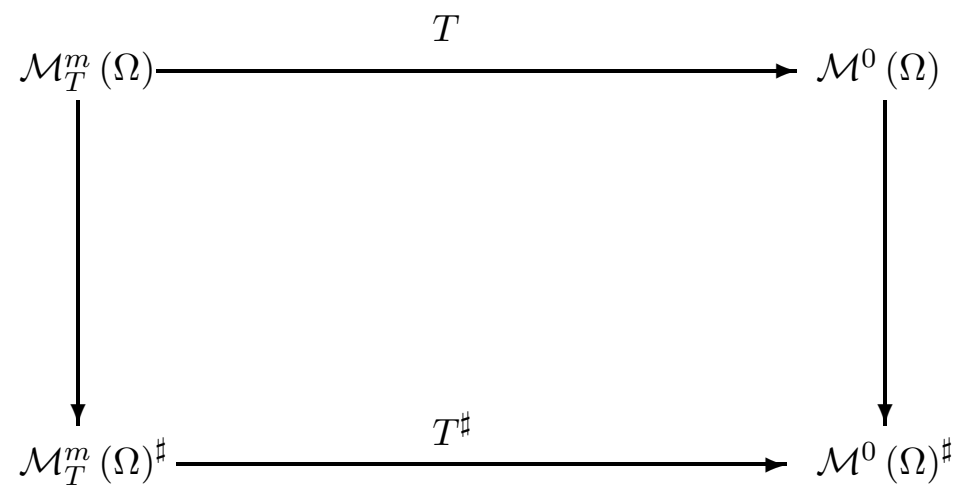

with $T^{\sharp}$ an order isomorphism. Hence, for every $f \in \mathcal{C}^{0}(\Omega)$, the generalized equation

$$
T^{\sharp} U=f
$$

has a unique solution in $\mathcal{M}_{T}^{m}(\Omega)^{\sharp}$.

\subsection{Extended Concepts of Topology}

The aim of this paper is to cast the Order Completion Method of Oberguggenberger and Rosinger within the framework of the traditional topological and function analytical method. In order to achieve this aim it is necessary to consider topological type structures that are more general than the usual Hausdorff-Kuratowski-Bourbaki concept of topology. Note that this need for generalized concepts of topology is not unique to the problem considered here, but appears frequently in analysis- even in the relatively simple setting of locally convex linear spaces. Indeed, recall that there is no topology on the topological dual $E^{*}$ of a locally convex space $E$ such that the simple evaluation mapping

$$
e v: E \times E^{*} \rightarrow \mathbb{R}
$$

defined through

$$
e v:\left(x, x^{*}\right) \mapsto\left\langle x^{*}, x\right\rangle
$$


$\mathrm{J} \mathrm{H}$ van der Walt

is jointly continuous, unless $E$ is a normable space. Other examples include some natural notions of convergence in measure theory and in the theory of ordered spaces, which can not be induced by a topology. For a more detailed motivation of the need for generalized concepts of topology we refer the reader to (Beattie and Butzmann, 2002) and (Rosinger and van der Walt, 2007).

The most general such topological type structures so far considered in the literature was introduced by Rosinger, see (Rosinger, 1963) through (Rosinger, 1965), and more recently (Rosinger and van der Walt, 2007). A highly particular case of these topological type structures are the celebrated convergence structures and convergence spaces, see (Beattie and Butzmann, 2002), which are defined in terms of convergent filters. The collection of convergence structures on a given set $X$ contains the collection of all topologies on $X$ so that Hausdorff-Kuratowsky-Bourbaki topology is a special case of convergence structures.

The theory of uniform spaces is a particular but highly important case of general topology, among others due to the issue of completeness and completion. The generalization of uniform spaces and uniformities within the setting of convergence spaces are uniform convergence structures and uniform convergence spaces. Whereas the uniform spaces are rather particular amongst topological spaces, almost all convergence spaces are uniform convergence space. Indeed, every Hausdorff convergence space is a uniform convergence space, and there are even some uniform convergence spaces that are not Hausdorff! As is the case for uniform spaces, every Hausdorff uniform convergence space admits a Hausdorff completion which is unique up to homeomorphism. For details on uniform convergence spaces we refer the reader to (Beattie and Butzmann, 2002), (Gähler, 1977), (Gähler, 1978) or Appendix A.

\subsection{Order Convergence and the Order Convergence Structure}

As mentioned, there are many natural and useful modes of convergence that can be defined in terms of a partial order $\leq$ on a set $X$, see for instance (Birkhoff, 1973), (Luxemburg and Zaanen, 1971) and (Peressini, 1967). One of the better known such modes of convergence is order convergence of sequences, which can be defined on an arbitrary poset $X$ through

$$
\left(x_{n}\right) \text { order converges to } x \in X \Leftrightarrow\left(\begin{array}{l}
\exists\left(\lambda_{n}\right),\left(\mu_{n}\right) \subset X: \\
\left(\lambda_{n}\right) \text { increases to } x: \\
\left(\mu_{n}\right) \text { decreases to } x: \\
n \in \mathbb{N} \Rightarrow \lambda_{n} \leq x_{n} \leq \mu_{n}
\end{array}\right)
$$

It is well know that order convergence of sequences can, in general, not be induced by a topology, that is, for an arbitrary poset $X$ there is no topology $\tau$ on $X$ such that the convergent sequences with respect to $\tau$ are exactly those sequences that order converge, see for instance (Anguelov and van der Walt, 2005). However, recently it was shown that for a $\sigma$-distributive lattice $X$ there is a convergence structure $\lambda$ on $X$ that induces order convergence of sequences, see (Anguelov and van der Walt, 2005) and (van der Walt, 2006). One such a convergence structure, the Order Convergence Structure $\lambda_{o}$, was defined as

$$
\mathcal{F} \in \lambda_{o}(x) \Leftrightarrow\left(\begin{array}{l}
\exists\left(\lambda_{n}\right),\left(\mu_{n}\right) \text { on } X: \\
\left(\lambda_{n}\right) \text { increases to } x: \\
\left(\mu_{n}\right) \text { decreases to } x: \\
\left\{\left[\lambda_{n}, \mu_{n}\right]: n \in \mathbb{N}\right\} \subseteq \mathcal{F}
\end{array}\right)
$$

Note that this result applies amongst others to all vector lattices. Moreover, if a vector lattice $X$ is also Archimedean, then the Order Convergence Structure is a vector space convergence structure in the sense of (Beattie and Butzmann, 2002). An important result obtained in (van der Walt, 2006) is that the completion of the uniform convergence 
structure induced on $X$ by $\lambda_{o}$ and the linear structure is the Dedekind $\sigma$-completion of $X$ equipped with the Order Convergence Structure. In case $X$ is order separable (Luxemburg and Zaanen, 1971), the completion is in fact the Dedekind completion of $X$.

Since the space $\mathcal{M}^{0}(\Omega)$ that appears in the Order Completion Method can be viewed as an order separable Archimedean vector lattice, the completion result described above appears to provide a way of casting the Order Completion Method in the framework of uniform convergence spaces. However, one of the main advantages of the Order Completion Method is that it makes no distinction between linear and nonlinear equations, as is usually the case for topological methods. The completion result for the Order Convergence Structure on an Archimedean vector lattice, on the other hand, is a distinctly linear result. However, in this regard it was recently shown (van der Walt, 2007 [1]) that the Order Convergence Structure on the order isomorphic copy $\mathcal{M L}^{0}(\Omega)$ of $\mathcal{M}^{0}(\Omega)$ is induced by a nontrivial uniform convergence structure, and the resulting completion is again the Dedekind completion $\mathbb{H}_{n f}(\Omega)$ of $\mathcal{M L}^{0}(\Omega)$. Here $\mathbb{H}_{n f}(\Omega)$ denotes the set of all nearly finite Hausdorff continuous functions, (Anguelov, 2004) and (Sendov, 1990).

\section{Spaces of Functions}

This section is devoted to a short introduction of the spaces of functions, and spaces of generalized functions on which the PDEs, and their extensions, are to act. As with the Order Completion Method, we start with the space $\mathcal{C}_{n d}^{m}(\Omega)$. On this space we can consider the Lower- and Upper Baire Operators

$$
\begin{aligned}
& I(f)(x)=\sup \left\{\inf \{z \in f(y): y \in V\}: V \in \mathcal{V}_{x}\right\}, x \in X \\
& S(f)(x)=\inf \left\{\sup \{z \in f(y): y \in V\}: V \in \mathcal{V}_{x}\right\}, x \in X
\end{aligned}
$$

where $\mathcal{V}_{x}=\{V \subseteq X \mid V$ a neighbourhood of $x\}$ is the neighborhood filter at $x \in \Omega$. These operators were first introduced by Baire (Baire, 1905) for real valued functions of a real variable, and later generalized by Sendov (Sendov, 1990) and Anguelov (Anguelov, 2004 ). Note that the natural setting of these operators, within the context of point valued functions, is the set $\mathcal{A}(\Omega)$ consisting of all functions $f$ that act on $\Omega$ and take values in the extended real line $\overline{\mathbb{R}}=\mathbb{R} \cup\{ \pm \infty\}$. There the operators $I$ and $S$ characterize semi-continuity. Indeed,

$$
\begin{aligned}
& f \in \mathcal{A}(\Omega) \text { is lower semi-continuous } \Leftrightarrow I(f)=f \\
& f \in \mathcal{A}(\Omega) \text { is upper semi-continuous } \Leftrightarrow S(f)=f
\end{aligned}
$$

Moreover, compositions of these operators characterize the normal semi-continuous functions, see (Dilworth, 1950) and (Anguelov, 2004):

$$
\begin{gathered}
f \in \mathcal{A}(\Omega) \text { is normal lower semi-continuous } \Leftrightarrow I(S(f))=f \\
f \in \mathcal{A}(\Omega) \text { is normal upper semi-continuous } \Leftrightarrow S(I(f))(x)=f
\end{gathered}
$$

The normal lower and upper semi-continuous functions were introduced in (Dilworth, 1950) in connection with his attempts to find the Dedekind order completion of spaces of continuous functions. This problem was finally solved by Anguelov, see (Anguelov, 2004), where he characterized the Dedekind completion of $\mathcal{C}(X)$ and some of its subspaces as spaces of Hausdorff continuous functions. 
We now consider the space

$$
\mathcal{M L}^{m}(\Omega)=\left\{I(S(f)): f \in \mathcal{C}_{n d}^{m}(\Omega)\right\} .
$$

That is, $\mathcal{M L}^{m}(\Omega)$ is the image of $\mathcal{C}_{n d}^{m}(\Omega)$ under the operator $I \circ S$. Since $(I \circ S)(f)(x)$ is equal to $f(x)$ whenever $f$ is continuous at $x$, it is $m$ times continuously differentiable everywhere except on a closed nowhere dense subset of $\Omega$. Hence it follows that $\mathcal{M L}^{m}(\Omega) \subset$ $\mathcal{C}_{n d}^{m}(\Omega)$. By the idempotence of the composite operator $I \circ S$, (Anguelov, 2004), it follows by (15) and (3) that $\mathcal{M L}^{m}(\Omega)$ consists of all normal lower semi-continuous functions which are $m$-times continuously differentiable everywhere except on some closed nowhere dense subset of $\Omega$. This space was introduced and studied in (van der Walt, 2007 [1]) and (van der Walt, $2007[2]$ ), and it was shown that $\mathcal{M L}^{m}(\Omega)$ is an order isomorphic copy of $\mathcal{M}^{m}(\Omega)$. Recall that $\mathcal{M}^{m}(\Omega)=\mathcal{C}_{n d}^{m}(\Omega) / \sim$, where $\sim$ is the equivalence relation $(5)$.

We now consider the family $\mathcal{J}_{o}$ of filters on the Cartesian product $\mathcal{M L}^{0}(\Omega) \times \mathcal{M L}^{0}(\Omega)$.

DEFINITION 1. A filter $\mathcal{U}$ on $\mathcal{M L}^{0}(\Omega) \times \mathcal{M L}^{0}(\Omega)$ belongs to the family $\mathcal{J}_{\text {o }}$ whenever there exists $k \in \mathbb{N}$ such that

$$
\begin{aligned}
& \forall i=1, \ldots, k: \\
& \exists\left(I_{n}^{i}\right) \text { nonempty order intervals : } \\
& \text { 1) } I_{n}^{i} \supseteq I_{n+1}^{i} \text { for every } n \in \mathbb{N} \\
& \text { 2) } \forall V \subseteq \Omega \text { open } \cap_{n \in \mathbb{N}} I_{n \mid V}^{i}=\emptyset \text { or there } \\
& \text { is } f_{i} \in \mathcal{M L}^{0}(V) \text { such that } \cap_{n \in \mathbb{N}} I_{n \mid V}^{i}=\left\{f_{i}\right\}
\end{aligned}
$$

and

$$
\left(\mathcal{I}^{1} \times \mathcal{I}^{1}\right) \cap \ldots \cap\left(\mathcal{I}^{k} \times \mathcal{I}^{k}\right) \subset \mathcal{U} \text { where } \mathcal{I}^{i}=\left[\left\{I_{n}^{i}: n \in \mathbb{N}\right\}\right]
$$

The family $\mathcal{J}_{o}$ is in fact a uniform convergence structure on $\mathcal{M L}^{0}(\Omega)$, which we call the Uniform Order Convergence Structure. It induces the Order Convergence Structure on $\mathcal{M L}^{0}(\Omega)$, see (van der Walt, 2007 [1]) and (van der Walt, 2007 [2]), and hence the following properties are immediate:

- A filter $\mathcal{F}$ on $\mathcal{M L}^{0}(\Omega)$ converges with respect to the induced convergence structure if and only if $\mathcal{F}$ converges in the Order Convergence Structure on $\mathcal{M L}^{0}(\Omega)$.

- A sequence $\left(f_{n}\right)$ on $\mathcal{M L}^{0}(\Omega)$ converges to $f \in \mathcal{M L}^{0}(\Omega)$ if and only if $\left(f_{n}\right)$ order converges to $f$.

- The uniform convergence space $\mathcal{M L}^{0}(\Omega)$ is uniformly Hausdorff.

We now consider a system of $K$ nonlinear PDEs, each of order at most $m$, in $K$ unknown functions $u_{1}, \ldots, u_{K}$

$$
\mathbf{T}(x, D) \mathbf{u}(x)=\mathbf{f}(x), x \in \Omega \subseteq \mathbb{R}^{n} \text { an open set. }
$$

Here $\mathbf{u}: \Omega \rightarrow \mathbb{R}^{K}$ is a $K$-dimensional vector valued function with components $u_{1}, \ldots, u_{K} \in$ $\mathcal{C}^{m}(\Omega)$. The righthand term $\mathbf{f}: \Omega \rightarrow \mathbb{R}^{K}$ is supposed to have continuous components $f_{1}, \ldots, f_{K}: \Omega \rightarrow \mathbb{R}$. The PDE-operator $\mathbf{T}(x, D)$ is defined through

$$
\mathbf{T}(x, D): \mathbf{u}(x) \mapsto \mathbf{F}\left(x, \ldots, u_{i}(x), \ldots, D^{\alpha} u_{i}, \ldots\right) \text { with } i=1, \ldots, K,|\alpha| \leq m
$$


where $\mathbf{F}: \Omega \times \mathbb{R}^{M} \rightarrow \mathbb{R}^{K}$ is a jointly continuous function in all of its arguments with components $F_{1}, \ldots, F_{K}: \Omega \times \mathbb{R}^{M} \rightarrow \mathbb{R}$. One can now write equation (21) in the form

$$
\begin{array}{ccc}
T_{1}(x, D) \mathbf{u}(x) & = & f_{1}(x) \\
\vdots & \vdots & \vdots
\end{array}, x \in \Omega .
$$

where each $T_{i}(x, D)$ is defined through

$$
T_{i}(x, D): \mathbf{u}(x) \mapsto F_{i}\left(x, \ldots, u_{j}(x), \ldots, D^{\alpha} u_{j}, \ldots\right) \text { with } j=1, \ldots, K
$$

With the operator $T(x, D)$ we associate a mapping

$$
\widetilde{\mathbf{T}}: \mathcal{M L}^{m}(\Omega)^{K} \rightarrow \mathcal{M L}^{0}(\Omega)^{K}
$$

with components

$$
\widetilde{T}_{i}: \mathcal{M L}^{m}(\Omega) \rightarrow \mathcal{M L}^{0}(\Omega)
$$

defined through

$$
\widetilde{T}_{i}(\mathbf{u}): x \rightarrow(I \circ S)\left(F\left(\cdot, u_{1}, \ldots, u_{K}, \ldots, \mathcal{D}^{\alpha} u_{i}, \ldots\right)\right)(x), x \in \Omega
$$

where

$$
\mathcal{D}^{\alpha} f=(I \circ S)\left(D^{\alpha} f\right), f \in \mathcal{C}_{n d}^{m}(\Omega) .
$$

Since each $\mathcal{D}^{\alpha} u_{i}$ is continuous on an open and dense set of $\Omega$, it follows by the continuity of the $F_{i}$ that $\widetilde{\mathbf{T}} \mathbf{u} \in \mathcal{M L}^{0}(\Omega)^{K}$ for every $\mathbf{u} \in \mathcal{M L}^{m}(\Omega)^{K}$. Moreover, since (13) and (14) imply that a function continuous at a point $x \in \Omega$ is invariant under the operators $I$ and $S$, at $x$, it follows that

$$
\begin{aligned}
& \forall \mathbf{u} \in \mathcal{M L}^{m}(\Omega): \\
& \exists \Gamma \subset \Omega:
\end{aligned}
$$

$\Gamma$ is closed nowhere dense :

$$
x \in \Omega \backslash \Gamma \Rightarrow \widetilde{\mathbf{T}}(\mathbf{u})(x)=\mathbf{T}(x, D) \mathbf{u}(x)
$$

On the space $\mathcal{M L}^{m}(\Omega)^{K}$ consider the equivalence relation induced by $\widetilde{\mathbf{T}}$ through

$$
\mathbf{u} \sim \widetilde{\mathbf{T}} \mathbf{v} \Leftrightarrow \widetilde{\mathbf{T}} \mathbf{u}=\widetilde{\mathbf{T}} \mathbf{v}
$$

We denote the quotient space $\mathcal{M} \mathcal{L}^{m}(\Omega)^{K} / \sim_{\widetilde{\mathbf{T}}}$ by $\mathcal{M} \mathcal{L}_{\widetilde{\mathbf{T}}}^{m}(\Omega)$. With the mapping $\widetilde{\mathbf{T}}$ we now associate a mapping

$$
\widehat{\mathbf{T}}: \mathcal{M L}_{\widetilde{\mathbf{T}}}^{m}(\Omega) \rightarrow \mathcal{M} \mathcal{L}^{0}(\Omega)^{K}
$$

which assigns to each equivalence class in $\mathcal{M L}_{\widetilde{\mathbf{T}}}^{m}(\Omega)$ its image under the operator $\widetilde{\mathbf{T}}$, that is,

$$
\begin{aligned}
& \forall \mathbf{U} \in \mathcal{M L}_{\widetilde{\mathbf{T}}}^{m}(\Omega): \\
& \forall \mathbf{u} \in \mathbf{U}: \\
& \widehat{\mathbf{T}}(\mathbf{U})=\widetilde{\mathbf{T}}(\mathbf{u})
\end{aligned}
$$

The generalized version of equation (21) is

$$
\widehat{\mathbf{T}} \mathbf{U}=\mathbf{f},
$$


where $\mathbf{U}$ is an equivalence class under the equivalence $\sim_{\widetilde{\mathbf{T}}}$. The mapping $\widehat{\mathbf{T}}$ is injective, but it is in general not surjective.

We consider the space $\mathcal{M L}^{0}(\Omega)^{K}$ equipped with the product uniform convergence structure when each copy of $\mathcal{M L}^{0}(\Omega)$ carries the Uniform Order Convergence Structure.

DEFINITION 2. A filter $\mathcal{U}$ on $\mathcal{M L}^{0}(\Omega)^{K} \times \mathcal{M L}^{0}(\Omega)^{K}$ belongs to $\mathcal{J}_{o}^{K}$ whenever $\left(\pi_{i} \times \pi_{i}\right)(\mathcal{U}) \in$

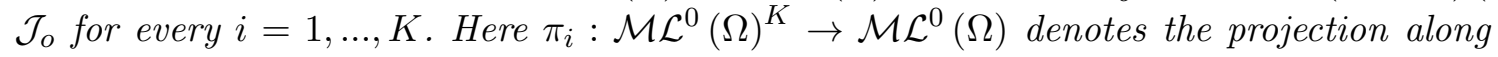
the ith coordinate.

$\mathcal{M L}_{\widetilde{\mathbf{T}}}^{m}(\Omega)$ carries the initial uniform convergence structure $\mathcal{J}_{\widetilde{\mathbf{T}}}$ with respect to the injective mapping

$$
\widehat{\mathbf{T}}: \mathcal{M} \mathcal{L}_{\widetilde{\mathbf{T}}}^{m}(\Omega) \rightarrow \mathcal{M} \mathcal{L}^{0}(\Omega)^{K}
$$

DEFINITION 3. A filter $\mathcal{U}$ on $\mathcal{M} \mathcal{L}_{\widetilde{\boldsymbol{T}}}^{m}(\Omega) \times \mathcal{M L}_{\widetilde{\boldsymbol{T}}}^{m}(\Omega)$ belongs to $\mathcal{J}_{\widetilde{\boldsymbol{T}}}$ whenever $(\widetilde{\boldsymbol{T}} \times \widetilde{\boldsymbol{T}})(\mathcal{U})$ belongs to $\mathcal{J}_{o}^{K}$.

From the injectivity of $\widehat{\mathbf{T}}$ we obtain

$$
\widehat{\mathbf{T}}: \mathcal{M L}_{\widetilde{\mathbf{T}}}^{m}(\Omega) \hookrightarrow \mathcal{M L}^{0}(\Omega)^{K} \text { is a uniformly continuous embedding }
$$

The completion of $\mathcal{M L}^{0}(\Omega)$ can be constructed as a set of Hausdorff continuous functions. In fact, it is the set $\mathbb{H}_{n f}(\Omega)$ consisting of all nearly finite Hausdorff continuous functions, equipped with a suitably chosen uniform convergence structure, see (van der Walt, 2007 [2]) for details. Hence it follows by Theorem 16 that the completion of $\mathcal{M L}^{0}(\Omega)^{K}$ is $\mathbb{H}_{n f}(\Omega)^{K}$, equipped with the product uniform convergence structure. Moreover, it now follows by (29) and Theorem 17 that the completion of $\mathcal{M L}_{\widetilde{\mathbf{T}}}^{m}(\Omega)$ is homeomorphic to a subspace $\mathbb{H}_{\widetilde{\mathbf{T}}}(\Omega)$ of $\mathbb{H}_{n f}(\Omega)$. We will not give details concerning Hausdorff continuous functions. For details concerning Hausdorff continuous functions, we refer the reader to (Anguelov, 2004).

\section{Approximation Results}

We now again consider a system of $K$ nonlinear PDEs of the form (21) through (22). Recall that the Order Completion Method for single nonlinear PDEs of the form (1) through (2) is based on the simple approximation result (4). This result is follows from the fact that every continuous function can be approximated locally by a sequence of polynomials. In this section we extend this result to the general $K$-dimensional case, for $K \geq 1$ arbitrary but given.

A natural assumption on the function $\mathbf{F}: \Omega \times \mathbb{R}^{M} \rightarrow \mathbb{R}^{K}$, and hence the PDE-operator $\mathbf{T}(x, D)$, and the righthand term $\mathbf{f}$ is that, for every $x \in \Omega$

$$
\mathbf{f}(x) \in \operatorname{int} R_{x} \text { where } R_{x}=\left\{\mathbf{F}\left(x, \xi_{1 \alpha}, \ldots, \xi_{i \alpha}, \ldots\right): \xi_{i \alpha} \in \mathbb{R}, i=1, \ldots, K,|\alpha| \leq m\right\}
$$

Note that (30) is is of a technical nature, and hardly a restriction on the class of PDEs considered. In fact, every linear PDE, and also most nonlinear PDEs, satisfy (30). It is necessary condition for the existence of a classical solution to (21) in a neighborhood of $x$.

THEOREM 4. Consider a system of PDEs of the form (21) through (22) that also satisfies (30). For every $\epsilon>0$ there exists a closed nowhere dense set $\Gamma_{\epsilon} \subset \Omega$ with zero Lebesgue measure, and a function $\boldsymbol{U}_{\epsilon} \in \mathcal{C}^{m}\left(\Omega \backslash \Gamma_{\epsilon}\right)^{K}$ with components $U_{\epsilon, 1}, \ldots, U_{\epsilon, K}$ such that

$$
f_{i}(x)-\epsilon \leq T_{i}(x, D) \boldsymbol{U}_{\epsilon}(x) \leq f_{i}(x), x \in \Omega \backslash \Gamma_{\epsilon}
$$




\section{Proof.}

Let

$$
\Omega=\bigcup_{\nu \in \mathbb{N}} C_{\nu}
$$

where, for $\nu \in \mathbb{N}$, the compact sets $C_{\nu}$ are $n$-dimensional intervals

$$
C_{\nu}=\left[a_{\nu}, b_{\nu}\right]
$$

with $a_{\nu}=\left(a_{\nu, 1}, \ldots, a_{\nu, n}\right), b_{\nu}=\left(b_{\nu, 1}, \ldots, b_{\nu, n}\right) \in \mathbb{R}^{n}$ and $a_{\nu, i} \leq b_{\nu, i}$ for every $i=1, \ldots, n$.We also assume that $C_{\nu}$, with $\nu \in \mathbb{N}$ are locally finite, that is,

$$
\forall x \in \Omega \exists V_{x} \subseteq \Omega \text { a neighborhood of } x:\left\{\nu \in \mathbb{N}: C_{\nu} \cap V_{x} \neq \emptyset\right\} \text { is finite }
$$

We also assume that the interiors of $C_{\nu}$, with $\nu \in \mathbb{N}$, are pairwise disjoint. We note that note that such $C_{\nu}$ exist, see (Forster, 1981).

Let us now take $\epsilon>0$ given arbitrary but fixed. Let us take $\nu \in \mathbb{N}$ and apply Lemma 5 to each $x_{0} \in C_{\nu}$. Then we obtain $\delta_{x_{0}}>0$ and $P_{x_{0}, 1}, \ldots, P_{x_{0}, K}$ polynomial in $x \in \mathbb{R}^{n}$ such that

$$
f_{i}(x)-\epsilon \leq T_{i}(x, D) \mathbf{P}_{x_{0}}(x) \leq f(x), x \in \Omega \cap \bar{B}\left(x_{0}, \delta_{x_{0}}\right) \text { and } i=1, \ldots, K
$$

where $\mathbf{P}_{x_{0}}: \mathbb{R}^{n} \rightarrow \mathbb{R}^{K}$ is the $K$-dimensional vector valued function with components $P_{x_{0}, 1}, \ldots, P_{x_{0}, K}$. Since $C_{\nu}$ is compact, it follows that

$$
\begin{aligned}
& \exists \delta>0: \\
& \forall x_{0} \in C_{\nu}: \\
& \exists P_{x_{0}, 1}, \ldots, P_{x_{0}, K} \text { polynomial in } x \in \mathbb{R}^{n}: \\
& \left\|x-x_{0}\right\| \leq \delta \Rightarrow f_{i}(x)-\epsilon \leq T_{i}(x, D) \mathbf{P}_{x_{0}}(x) \leq f(x), x \in \bar{B}\left(x_{0}, \delta\right) \cap \Omega
\end{aligned}
$$

where $i=1, \ldots, K$. Subdivide $C_{\nu}$ into $n$-dimensional intervals $I_{\nu, 1}, \ldots, I_{\nu, \mu}$ with diameter not exceeding $\delta$ such that their interiors are pairwise disjoint. If $a_{j}$ with $j=1, \ldots, \mu$ is the center of the interval $I_{\nu, j}$ then by (36) there exists $P_{a_{j}, 1}, \ldots, P_{a_{j}}, K$ polynomial in $x \in \mathbb{R}^{n}$ such that

$$
f_{i}(x)-\epsilon \leq T_{i}(x, D) \mathbf{P}_{a_{j}}(x) \leq f_{i}(x), x \in I_{\nu, j}
$$

where $i=1, \ldots, K$. Now set

$$
\Gamma_{\nu, \epsilon}=C_{\nu} \backslash\left(\left(\bigcup_{j=1}^{\mu} \operatorname{int} I_{\nu, j}\right) \cup \operatorname{int} C_{\nu}\right)
$$

that is, $\Gamma_{\nu, \epsilon}$ is a rectangular grid generated as a finite union of hyperplanes. Furthermore, using (37), we find

$$
\mathbf{U}_{\nu, \epsilon} \in \mathcal{C}^{m}\left(C_{\nu} \backslash \Gamma_{\nu, \epsilon}\right)
$$

such that

$$
f_{i}(x)-\epsilon \leq T_{i}(x, D) \mathbf{U}_{\nu, \epsilon}(x) \leq f_{i}(x), x \in C_{\nu} \backslash \Gamma_{\nu, \epsilon}
$$

In view of (34) it follows that

$$
\Gamma_{\epsilon}=\bigcup_{\nu \mathbb{N}} \Gamma_{\nu, \epsilon} \text { is closed nowhere dense and mes }\left(\Gamma_{\epsilon}\right)=0
$$

From (32), (39) and (40) we obtain (31).

The above proof relies on the following lemma which is in fact the basic approximation result. 
LEMMA 5. Consider a system of PDEs of the form (21) through (22) that also satisfies (30). Then

$$
\begin{aligned}
& \forall x_{0} \in \Omega, \epsilon>0: \\
& \exists \delta>0, P_{1}, \ldots, P_{K} \text { polynomial in } x \in \mathbb{R}^{n}: \\
& x \in B\left(x_{0}, \delta\right) \cap \Omega, 1 \leq i \leq k \Rightarrow f_{i}(x)-\epsilon \leq T_{i}(x, D) \boldsymbol{P}(x) \leq f_{i}(x)
\end{aligned}
$$

Here $\boldsymbol{P}$ is the $K$-dimensional vector valued function with components $P_{1}, \ldots, P_{K}$.

Proof.

For any $x_{0} \in \Omega$ and $\epsilon>0$ small enough it follows by (30) that there exist

$$
\xi_{i \alpha} \in \mathbb{R} \text { with } i=1, \ldots, K \text { and }|\alpha| \leq m
$$

such that

$$
F_{i}\left(x_{0}, \ldots, \xi_{i \alpha}, \ldots\right)=f_{i}\left(x_{0}\right)-\frac{\epsilon}{2}
$$

Now take $P_{1}, \ldots, P_{K}$ polynomials in $x \in \mathbb{R}^{n}$ that satisfy

$$
D^{\alpha} P_{i}\left(x_{0}\right)=\xi_{i \alpha} \text { for } i=1, \ldots, K \text { and }|\alpha| \leq m
$$

Then it is clear that

$$
T_{i}(x, D) \mathbf{P}\left(x_{0}\right)-f_{i}\left(x_{0}\right)=-\frac{\epsilon}{2}
$$

where $\mathbf{P}$ is the $K$-dimensional vector valued function on $\mathbb{R}^{n}$ with components $P_{1}, \ldots, P_{K}$. Hence (42) follows by the continuity of the $f_{i}$ and the $F_{i}$.

\section{Existence of Generalized Solutions}

We now proceed to establish both the existence and uniqueness of generalized solutions to the generalized equation (27). This result appears as an application of the theory developed in Sections 2 and 3, and the results concerning completions of uniform convergence spaces contained in Appendix A.

The uniqueness of the solution should not be interpreted as implying that any meaningful solutions are disregarded. Indeed, recall that the elements of the space $\mathcal{M} \mathcal{L}_{\widetilde{\mathbf{T}}}^{m}(\Omega)$ are equivalence classes under the equivalence (24). Hence, if there is, for instance, a solution $\mathbf{U} \in \mathcal{M} \mathcal{L}_{\widetilde{\mathbf{T}}}^{m}(\Omega)$, then $\mathbf{U}$ is the equivalence class containing all possible solutions $\mathbf{u} \in \mathcal{M L}^{m}(\Omega)^{K}$ to $(21)$.

Consider now the following commutative diagram:

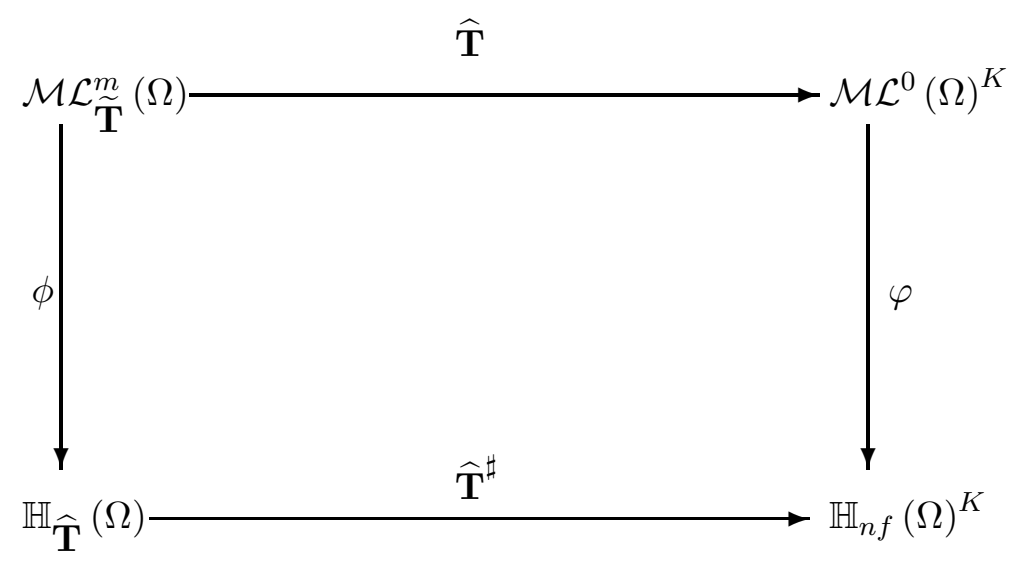


Here $\phi$ and $\varphi$ are the uniformly continuous embeddings associated with the completions $\mathbb{H}_{\widehat{\mathbf{T}}}(\Omega)$ and $\mathbb{H}_{n f}(\Omega)$, and $\widehat{\mathbf{T}}^{\sharp}$ is the extension of $\widehat{\mathbf{T}}$ achieved through uniform continuity. The existence of generalized solutions will follow from Theorem 4 and (29).

THEOREM 6. For every $\boldsymbol{f} \in \mathcal{C}^{0}(\Omega)^{K}$ that satisfies (30), there exists a unique $\boldsymbol{U} \in$ $\mathbb{H}_{\widetilde{\boldsymbol{T}}}(\Omega)^{K}$ such that

$$
\widehat{T}^{\sharp} U=f
$$

Proof. First let us show existence. For every $n \in \mathbb{N}$, Theorem 4 yields a closed nowhere dense set $\Gamma_{n} \subset \Omega$ and a function $\mathbf{u}_{n} \in \mathcal{C}^{m}(\Omega)$ that satisfies

$$
x \in \Omega \backslash \Gamma_{n} \Rightarrow f_{i}(x)-\frac{1}{n} \leq T_{i}(x, D) \mathbf{u}_{n}(x) \leq f_{i}(x), i=1, \ldots, K
$$

Since $\Gamma_{n}$ is closed nowhere dense we associate $\mathbf{u}_{n}$ with a function $\mathbf{v}_{n} \in \mathcal{M} \mathcal{L}^{m}(\Omega)$ in a unique way. Indeed, consider for instance the function

$$
\mathbf{w}_{n}: x \mapsto \begin{cases}\mathbf{u}_{n}(x) & \text { if } x \in \Omega \backslash \Gamma \\ 0 & \text { if } x \in \Gamma\end{cases}
$$

Now let $\mathbf{v}_{n}$ be the $K$-dimensional vector valued function with components $v_{n}^{i}=(I \circ S)\left(w_{n}^{i}\right)$. Denote by $\mathbf{V}_{n}$ the equivalence class generated by $\mathbf{v}_{n}$ under the equivalence relation $\sim_{\widetilde{\mathbf{T}}}$. Applying (23), (26) and (48) one finds that the sequence $\left(\widehat{\mathbf{T}}\left(\mathbf{V}_{n}\right)_{i}\right)$ is increasing in $\mathcal{M L}^{0}(\Omega)$ and has upper bound $f_{i}$, for each $i=1, \ldots, K$. It is clear that for each $i=1, \ldots, K$, $f_{i}$ is in fact the least upper of the sequence $\left(\widehat{\mathbf{T}}\left(\mathbf{V}_{n}\right)_{i}\right)$. Hence each $\left(\widehat{\mathbf{T}}\left(\mathbf{V}_{n}\right)_{i}\right)$ converges to $f_{i}$ in $\mathcal{M L}^{0}(\Omega)$ so that the sequence $\left(\widehat{\mathbf{T}}\left(\mathbf{V}_{n}\right)\right)$ converges to $\mathbf{f}$ in $\mathcal{M L}^{0}(\Omega)^{K}$. It now follows that $\left(\mathbf{V}_{n}\right)$ is a Cauchy sequence in $\mathcal{M} \mathcal{L}_{\widetilde{\mathbf{T}}}^{m}(\Omega)$ so that there exists $\mathbf{U} \in \mathbb{H}_{\widehat{\mathbf{T}}}(\Omega)$ that satisfies (47).

Since the mapping $\widehat{\mathbf{T}}: \mathcal{M L}_{\widetilde{\mathbf{T}}}^{m}(\Omega) \rightarrow \widehat{\mathbf{T}}$ is a uniformly continuous embedding by (29), the uniqueness of the solution $\mathbf{U}$ found above now follows by Theorem 18 .

\section{Conclusion}

It has been shown that the Order Completion Method for systems of nonlinear PDEs can be naturally considered within the framework of uniform convergence spaces. With respect to the previous existence and regularity results obtained by the Order Completion Method, we have not made any improvement. The class of systems of nonlinear PDEs treated is the same as that considered in (Oberguggenberger and Rosinger, 1994), that is, we considered all continuous PDEs.

However, the generalized solutions obtained are elements of the completion of a space of equivalence classes of piecewise smooth functions, which are assimilated with Hausdorff continuous functions. In order to obtain meaningful solutions, for instance classical solutions, a great deal of hard analysis is required. Within the framework of uniform convergence spaces as applied here, we have a sound topological foundation at our disposal. 


\section{Appendix}

\section{A. Convergence Structures}

A filter on a set $X$ is a nonempty collection $\mathcal{F}$ of nonempty subsets of $X$ that is closed under the formation of supersets and finite intersection. For any $x \in X$

$$
[x]=\{F \subseteq X: x \in F\}
$$

is the filter generated by $x$. A convergence structure on $X$ is now defined as follows.

DEFINITION 7. A convergence structure on $X$ is a mapping $\lambda$ from $X$ into the powerset of the set of all filters on $X$ that satisfies

$$
\begin{aligned}
& \text { (1) }[x] \in \lambda(x) \text { for every } x \in X \\
& \text { (2) If } \mathcal{F}, \mathcal{G} \in \lambda(x) \text {, then } \mathcal{F} \cap \mathcal{G} \in \lambda(x) \\
& \text { (3) If } \mathcal{F} \in \lambda(x) \text { and } \mathcal{F} \subseteq \mathcal{G} \text { then } \mathcal{G} \in \lambda(x)
\end{aligned}
$$

The pair $(X, \lambda)$ is called a convergence space.

A convergence space $X=(X, \lambda)$ is called Hausdorff if it satisfies

$$
x \neq y \Rightarrow \lambda(x) \cap \lambda(y)=\emptyset
$$

A convergence structure $\lambda$ on $X$ induces convergence of sequences through

$$
\left(x_{n}\right) \text { converges to } x \in X \Leftrightarrow\left\langle\left(x_{n}\right)\right\rangle=\left[\left\{\left\{x_{n}: n \geq k\right\}: k \in \mathbb{N}\right\}\right] \in \lambda(x)
$$

One can construct new convergence structures from old ones in the following way.

DEFINITION 8. Let $\left(X_{i}\right)_{i \in I}$ be a family of convergence spaces, $X$ a set and for every $i \in I$ let $f_{i}: X \rightarrow X_{i}$ be a mapping. A filter $\mathcal{F}$ on $X$ converges to $x \in X$ with respect to the initial convergence structure $\lambda$ on $X$ with respect to $\left(f_{i}\right)_{i \in I}$ whenever

$$
f_{i}(\mathcal{F}) \in \lambda_{i}(x) \text { for every } i \in I
$$

This construction leads, as a particular case, to the product and subspace convergence structures.

In order to define a uniform convergence structure we recall some notation. For subsets $U$ and $V$ of $X \times X$

$$
U^{-1}=\{(x, y) \in X \times X:(y, x) \in U\}
$$

and the composition of $U$ and $V$ is given by

$$
U \circ V=\left\{(x, y) \in X \times X: \begin{array}{l}
\exists z \in X: \\
(x, z) \in V,(z, y) \in U
\end{array}\right\}
$$

If $F \subseteq X$ then

$$
U[F]=\left\{x \in X: \begin{array}{l}
\exists y \in X \\
(y, x) \in U
\end{array}\right\}
$$

If $x \in X$, then one sets

$$
U[x]=U[\{x\}]
$$


For filters $\mathcal{U}$ and $\mathcal{V}$ we set

$$
\mathcal{U}^{-1}=\left\{U^{-1}: U \in \mathcal{U}\right\}
$$

and

$$
\mathcal{U} \circ \mathcal{V}=[\{U \circ V: U \in \mathcal{U}, V \in \mathcal{V}\}]
$$

whenever $U \circ V \neq \emptyset$ for every $U \in \mathcal{U}$ and $V \in \mathcal{V}$. If $\mathcal{F}$ is a filter on $X$, then

$$
\mathcal{U}[\mathcal{F}]=[\{U[F]: U \in \mathcal{U}, F \in \mathcal{F}\}]
$$

provided that $U[F] \neq \emptyset$ for every $U \in \mathcal{U}$ and $F \in \mathcal{F}$.

A uniform convergence structure on $X$ is a family $\mathcal{J}$ of filters on $X \times X$, as apposed to a uniformity on $X$ which consists of a single filter on $X \times X$.

DEFINITION 9. Let $X$ be a set. A family $\mathcal{J}$ of filters on $X \times X$ is called a uniform convergence structure on $X$ and $(X, \mathcal{J})$ a uniform convergence space if the following hold:

(1) $[x] \times[x] \in \mathcal{J}$ for all $x \in X$.

(2) $\mathcal{U} \cap \mathcal{V} \in \mathcal{J}$ whenever $\mathcal{U}, \mathcal{V} \in \mathcal{J}$.

(3) If $\mathcal{U} \in \mathcal{J}$ then $\mathcal{V} \in \mathcal{J}$ for every filter $\mathcal{V}$ on $X \times X$ such that $\mathcal{V} \supseteq \mathcal{U}$.

(4) If $\mathcal{U} \in \mathcal{J}$ then $\mathcal{U}^{-1} \in \mathcal{J}$.

(5) For all $\mathcal{U}, \mathcal{V} \in \mathcal{J}$ one has $\mathcal{U} \circ \mathcal{V} \in \mathcal{J}$ whenever the composition $\mathcal{U} \circ \mathcal{V}$ exists.

A uniform convergence structure $\mathcal{J}$ on $X$ induces a convergence structure $\lambda_{\mathcal{J}}$, the induced convergence structure, on $X$ through

$$
\mathcal{F} \in \lambda_{\mathcal{J}}(x) \Leftrightarrow[x] \times \mathcal{F} \in \mathcal{J}
$$

We can construct new uniform convergence structures from old ones in the following way:

DEFINITION 10. Let $X$ be a set and $\left(X_{i}\right)_{i \in I}$ a family of uniform convergence spaces, and for each $i \in I$ let $f_{i}: X \rightarrow X_{i}$ be a mapping. The initial uniform convergence structure $\mathcal{J}$ on $X$ with respect to $\left(f_{i}\right)_{i \in I}$ is defined through

$$
\mathcal{U} \in \mathcal{J} \Leftrightarrow\left(f_{i} \times f_{i}\right)(\mathcal{U}) \in \mathcal{J}_{i}
$$

This general construction yields amongst others the product and subspace uniform convergence structures. The product convergence structure on the Cartesian product $X$ of uniform convergence spaces $\left(X_{i}\right)_{i \in I}$ is the initial uniform convergence structure with respect to the projection mappings $\left(\pi_{i}: X \rightarrow X_{i}\right)$. The subspace uniform convergence structure on a subset $Y$ of a uniform convergence structure $X$ is the initial uniform convergence structure with respect to the inclusion mapping $\iota: Y \rightarrow X$. The following result (Beattie and Butzmann, 2002) is useful.

PROPOSITION 11. Let $X$ carry the initial uniform convergence structure with respect to $\left(f_{i}: X \rightarrow X_{i}\right)$. Then the induced convergence structure is the initial convergence structure with respect to the induced convergence structures on the $X_{i}$.

Some important classes of mappings between uniform convergence spaces are the following.

DEFINITION 12. Let $f: X \rightarrow Y$ be a mapping from the uniform convergence space $(X, \mathcal{J})$ into the uniform convergence space $\left(Y, \mathcal{J}^{\prime}\right)$.

(1) Then $f$ is uniformly continuous if $(f \times f)(\mathcal{U}) \in \mathcal{J}^{\prime}$ whenever $\mathcal{U} \in \mathcal{J}$

(2) The mapping $f$ is a uniform homeomorphism if $f$ is bijective and uniformly continuous with a uniformly continuous inverse.

(3) The mapping $f$ is an embedding if it is a uniform homeomorphism onto its range, that is, $f: X \rightarrow f(X) \subseteq Y$ is a homeomorphism when $f(X) \subseteq Y$ is considered with the subspace uniform convergence structure. 
With the concept of a uniform convergence space comes that of Cauchy filter, completeness and completion.

DEFINITION 13. Let $X$ be a uniform convergence space.

(1) A filter $\mathcal{F}$ on $X$ is a Cauchy filter if $\mathcal{F} \times \mathcal{F} \in \mathcal{J}$.

(2) $X$ is complete if every Cauchy filter on $X$ converges.

(3) A uniform convergence space $\tilde{X}$ is a completion of $X$ if there is a uniformly continuous embedding $\iota: X \rightarrow \widetilde{X}$ such that $\iota(X)$ is dense in $\widetilde{X}$ and, for every complete uniform convergence space $Y$ and uniformly continuous mapping $f: X \rightarrow Y$ there is a unique uniformly continuous mapping $f^{*}$ such that the diagram commutes.

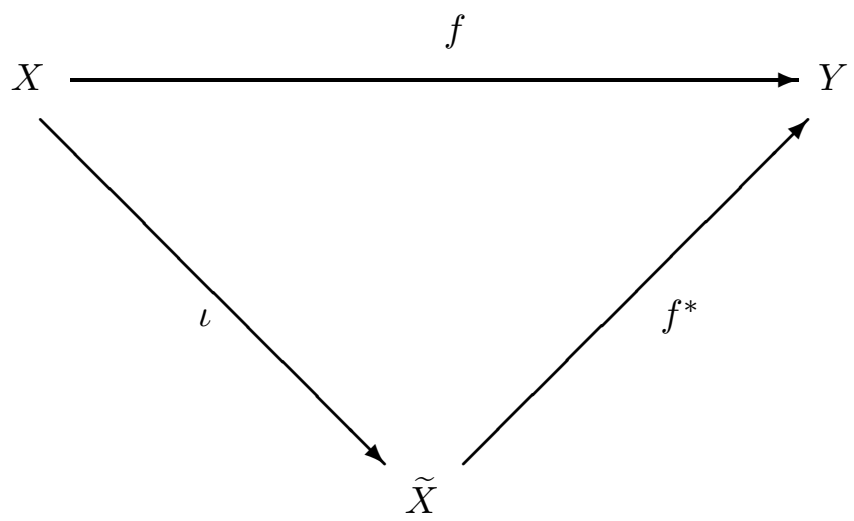

The main result concerning completions is the following (Gähler, 1978).

THEOREM 14. Let $X$ be a Hausdorff uniform convergence space. Then there exists a uniform convergence space $\widetilde{X}$ that satisfies Definition 13 (3). Moreover, $\widetilde{X}$ is unique up to homeomorphism.

The following result, found in (Gähler, 1978) and (Beattie and Butzmann, 2002), concerns the completeness of a product space.

THEOREM 15. Let $\left(X_{i}\right)_{i \in I}$ be a family of complete uniform convergence spaces. Then the product uniform convergence structure on $\prod_{i \in I} X_{i}$ is complete.

We now prove three results concerning completions.

THEOREM 16. Let $\left(X_{i}\right)_{i \in I}$ be a family of uniform convergence spaces and let $X$ denote their Cartesian product equipped with the product uniform convergence structure. Then the completion $\tilde{X}$ of $X$ is the product of the completions $\tilde{X}_{i}$ of the $X_{i}$.

Proof. First note that $\prod_{i \in I} X_{i}$ is complete by Theorem 15 . For every $i$, let $\varphi_{i}: X_{i} \rightarrow \tilde{X}_{i}$ be the uniformly continuous embedding associated with the completion $\widetilde{X}_{i}$ of $X_{i}$. Define the mapping $\varphi: X \rightarrow \prod \widetilde{X}_{i}$ through

$$
\varphi: x=\left(x_{i}\right) \mapsto\left(\varphi_{i}\left(x_{i}\right)\right)
$$

Moreover, for each $i$, let $\pi_{i}: X \rightarrow X_{i}$ be the projection mapping.

Since each $\varphi_{i}$ is injective, so is $\varphi$. Moreover, we have

$$
\mathcal{U} \in \mathcal{J}_{P} \Rightarrow\left(\pi_{i} \times \pi_{i}\right)(\mathcal{U}) \in \mathcal{J}_{i}
$$




$$
\begin{aligned}
& \Rightarrow\left(\varphi_{i} \times \varphi_{i}\right)\left(\left(\pi_{i} \times \pi_{i}\right)(\mathcal{U})\right) \in \widetilde{\mathcal{J}}_{i} \\
& \Rightarrow \prod_{i \in I}\left(\varphi_{i} \times \varphi_{i}\right)\left(\left(\pi_{i} \times \pi_{i}\right)(\mathcal{U})\right) \in \widetilde{\mathcal{J}}_{P} \\
& \Rightarrow(\varphi \times \varphi)(\mathcal{U}) \in \widetilde{\mathcal{J}}_{P}
\end{aligned}
$$

Hence $\varphi$ is uniformly continuous. Similarly, if the filter $\mathcal{V}$ on $\varphi(X) \times \varphi(X)$ belongs to the subspace uniform convergence structure, then

$$
\begin{aligned}
\left(\pi_{i} \times \pi_{i}\right)(\mathcal{V}) \in \widetilde{\mathcal{J}}_{i} & \Rightarrow\left(\varphi_{i}^{-1} \times \varphi_{i}^{-1}\right)\left(\left(\pi_{i} \times \pi_{i}\right)(\mathcal{V})\right) \in \mathcal{J}_{i} \\
& \Rightarrow \prod_{i \in I}\left(\varphi_{i}^{-1} \times \varphi_{i}^{-1}\right)\left(\left(\pi_{i} \times \pi_{i}\right)(\mathcal{V})\right) \in \mathcal{J}_{P} \\
& \Rightarrow\left(\varphi^{-1} \times \varphi^{-1}\right)(\mathcal{V}) \in \mathcal{J}_{P}
\end{aligned}
$$

so that $\varphi^{-1}$ is uniformly continuous. Hence $\varphi$ is a uniformly continuous embedding.

The denseness of $\varphi(X)$ in $\prod_{i \in I} \widetilde{X}_{i}$ follows by the denseness of $\varphi_{i}\left(X_{i}\right)$ in $\widetilde{X}_{i}$, for each $i \in I$. The extension property of uniformly continuous mappings into a complete uniform convergence space follows in the standard way.

THEOREM 17. Let $X$ be a subspace of the uniform convergence space $Y$. Then there is a subspace $\widetilde{X}$ of the completion $\widetilde{Y}$ of $Y$ such that $\widetilde{X}$ is the completion of $X$.

Proof. Let $\varphi: Y \rightarrow \widetilde{Y}$ be the uniformly continuous embedding associated with the completion $\tilde{Y}$ of $Y$. Set

$$
\widetilde{X}=\{\widetilde{y} \in \tilde{Y}: \exists \mathcal{F} \text { on } X, \varphi(\mathcal{F}) \text { converges to } \widetilde{y}\}
$$

Clearly $\varphi(X)$ is dense in $\tilde{X}$. To see that $\tilde{X}$ is complete, it suffices to show that it is a closed subspace of $\widetilde{Y}$. So let the filter $\mathcal{G}$, with a trace on $\widetilde{X}$, converge to $\widetilde{y} \in \widetilde{Y}$. The the filter

$$
\mathcal{F}=\left[\left\{\varphi^{-1}(G \cap \tilde{X}): G \in \mathcal{G}\right\}\right]
$$

satisfies

$$
\varphi(\mathcal{G}) \text { converges to } \widetilde{y}
$$

so that $\widetilde{y} \in \tilde{X}$. Hence $\widetilde{X}$ is a closed subspace of $\tilde{Y}$.

The extension property for uniformly continuous mappings $f: X \rightarrow Z$, where $Z$ is a complete uniform convergence space, follows in the standard way.

Lastly, we have the following.

THEOREM 18. Let $X$ and $Y$ be uniform convergence spaces, and $T: X \rightarrow Y$ a uniformly continuous embedding. Then there exists a uniformly continuous embedding $\widetilde{T}: \widetilde{X} \rightarrow \widetilde{Y}$, where $\widetilde{X}$ and $\widetilde{Y}$ are the completions of $X$ and $Y$ respectively, which extends $T$.

Proof. Since $X$ is homeomorphic to the subspace $T(X)$ of $Y$, it follows that $\tilde{X}$ is homeomorphic to $\widetilde{T(X)}$ which is a subspace of $\widetilde{Y}$ by Theorem 17 . Hence we obtain the following two commutative diagrams. 

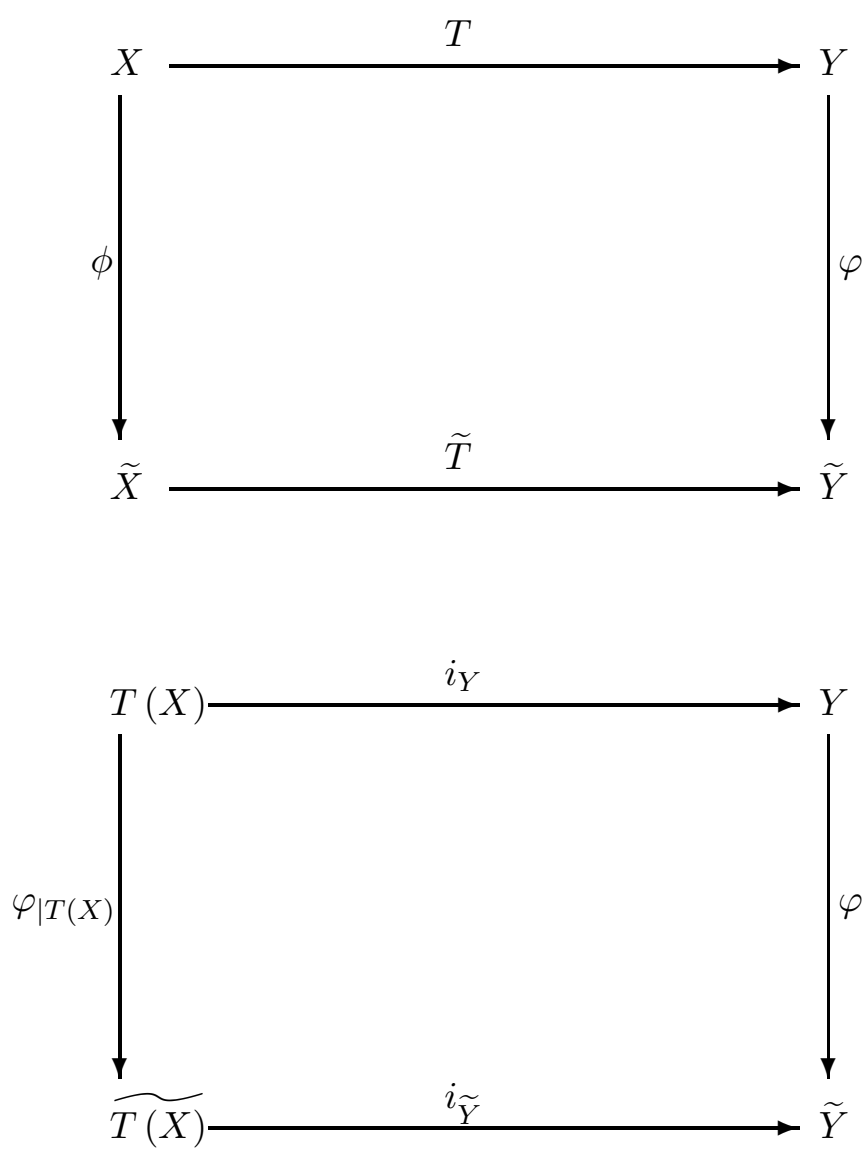

Here $i_{Y}$ and $i_{\widetilde{Y}}$ denotes the inclusion mappings on $Y$ and $\tilde{Y}$, respectively. It now follows that $\widetilde{T}: \widetilde{X} \rightarrow \widetilde{Y}$ is an embedding, which completes the proof.

\section{References}

Anguelov, R. Dedekind order completion of $\mathrm{C}(\mathrm{X})$ by Hausdorff continuous functions, Quaestiones Mathematicae 27, 153-170 (2004).

Anguelov, R., Markov, S. and Sendov, B. The Set of Hausdorff Continuous Functions - the Largest Linear Space of Interval Functions, Reliable Computing 12, 337-363 (2006).

Anguelov, R. and Rosinger E. E. Hausdorff Continuous Solutions of Nonlinear PDEs through the Order Completion Method, Quaestiones Mathematicae, Quaestiones Mathematicae 28(3), 271-285 (2005).

Anguelov, R. and van der Walt J. H. Order convergernce on $\mathcal{C}(X)$, Quaestiones Mathematicae 28(4), 425-457 (2005).

Arnold, V. I. Lectures on PDEs, Springer Universitext, (2004).

Baire, R. Lecons sur les fonctions discontinues, Collection Borel, Paris, (1905).

Beattie, R. and Butzmann H.-P. Convergence structures and applications to functional analysis, Kluwer Academic Plublishers, Dordrecht, Boston, London, (2002).

Birkhoff, G. Lattice Theory, AMS, Providence, Rhode Island, (1973).

Dilworth, R. P. The normal completion of the lattice of continuous functions, Trans. AMS (1950), 427-438.

Forster, O. Analysis 3, Integralrechnung im $\mathbb{R}^{n}$ mit Anwendungen, Friedr. Vieweg, Braunschweig, Wiesbaden, (1981).

Gähler, W. Grundstrukturen der anlysis I, Birkhäuser Verlag, Basel, (1977).

Gähler, W. Grundstrukturen der anlysis II, Birkhäuser Verlag, Basel, (1978).

Kriegl, A. and Michor P. W. The convenient Setting of Global Analysis, Mathematical Surveys Monographs, Vol. 53, AMS, Providence, (1997). 
Neuberger, J. W. Sobolev gradients and differential equations, Springer Lecture Notes in Mathematics vol. 1670, (1997).

Neuberger, J. W. Continuous Newton's method for polynomials, Mathematical Intelegencer 21, 18-23 (1999).

Neuberger, J. W. A near minimal hypothesis Nash-Moser theorem, Int. J. Pure Appl. Math. 4, 269-280 (2003)

Neuberger, J. W. Prospects of a central theory of partial differential equations 27(3), 47-55 (2005).

Luxemburg, W. A. and Zaanen A. C. Riesz Spaces I, North-Holland, Amsterdam London, (1971).

Markov, S. Calculus for interval functions of a real variable, Computing 22, 325-337 (1979).

Oberguggenberger, M. B. and Rosinger, E. E. Solution of continuous nonlinear PDEs through order completion, North-Holland, Amsterdam, London, New York, Tokyo, (1994).

Olejček, V. Order convergence and order topology on a poset, Int. J. of Theoretical Physics 38(2), 557-561 (1999)

Peressini, A. Ordered topological vector spaces, Harper \& Row, New York, Evanston, London, (1967).

Rosinger, E. E. Pseudotopological structures, Stud. Cerc. Mat. 14(2), 223-251 (1963).

Rosinger, E. E. Pseudotopological structures II, Stud. Cerc. Mat. 16(9), 1085-1110 (1964).

Rosinger, E. E. Pseudotopological structures III, Stud. Cerc. Mat. 17(7), 1133-1143 (1965).

Rosinger, E. E. New Method for solving large classes of systems of nonlinear PDEs, arXiv:math.GM/0610279

Rosinger, E. E. and van der Walt, J. H. Beyond topology, to appear.

Sendov, B. Hausdorff approximations, Kluwer Academic, Boston (1990).

van der Walt, J. H. Order convergence in sets of Hausdorff continuous functions. Honors Essay, University of Pretoria (2004).

van der Walt, J. H. Order convergence on Archimedean vector lattices. MSc Thesis, University of Pretoria (2006).

van der Walt, J. H. Uniform convergence structures on function spaces, to appear.

van der Walt, J. H. Uniform Order Convergence Structure on $\mathcal{M L}^{0}(\Omega)$, to appear. 
